\title{
Synthesis and properties of hectorite/poly(AM/IA) nanocomposite hydrogels with high gel strength
}

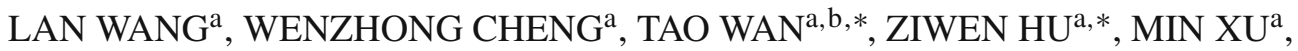 \\ RUIXIANG LI $^{\mathrm{a}}$ and CHUZHANG ZOU ${ }^{\mathrm{a}}$ \\ ${ }^{a}$ Mineral Resources Chemistry Key Laboratory of Sichuan Higher Education Institutions, Chengdu University \\ of Technology, Chengdu 610059, China \\ ${ }^{\mathrm{b}}$ State Key Lab of Geohazard Prevention and Geoenvironment Protection, Chengdu University of Technology, \\ Chengdu 610059, China \\ e-mail: wanglan@cdut.edu.cn; wantaos@126.com; huziwen@cdut.cn
}

MS received 11 September 2014; revised 28 October 2014; accepted 30 October 2014

\begin{abstract}
A novel hectorite/poly(AM/IA)nanocomposite hydrogel was synthesized by inverse microemulsion polymerization. The influence of hectorite amount on water absorbency rate, gel strength and shearresistance was investigated. Dynamic viscoelasticity behaviour of the nanocomposite hydrogels was also studied. The nanocomposite hydrogels showed suitable water absorbency and shear-resistance, high gel strength, solid-like behaviour in the whole oscillation frequency region and enhanced viscoelastic behaviours under high stress. TEM indicated that the as-synthesized hydrogel particles were regular and spherical in shape with an average particle size of $43 \mathrm{~nm}$ in the range of 30-65 $\mathrm{nm}$.
\end{abstract}

Keywords. Hydrogel; hectorite; nanocomposite; microemulsion polymerization.

\section{Introduction}

Superabsorbent hydrogels (SAHs) have been widely used in many fields such as hygienic products, ${ }^{1}$ agriculture, ${ }^{2,3}$ waste water treatment, ${ }^{4,5}$ drug-delivery systems $^{6-8}$ and enhanced oil recovery. ${ }^{9}$ However, superabsorbent hydrogels made from either natural or synthetic sources are limited in their industrial and biomedical applications due to their poor mechanical properties caused by irregularly distributed crosslinking. Therefore, in recent years, many efforts have focused on enhancing the mechanical strength of superabsorbent hydrogels. ${ }^{10,11}$ Nanocomposite hydrogels (NC gels), consisting of immiscible organic and inorganic components and complex nanometer-scale structures, exhibited extraordinary mechanical and optical properties which could overcome the limitations of conventional chemically cross-linked hydrogels. ${ }^{12-15}$

Laponite consists of synthetic silicate nanoparticles with an average diameter of $30 \mathrm{~nm}$ and a thickness of $1 \mathrm{~nm}$. Contrary to the conventional chemically crosslinked hydrogels, the NC gels formed using Laponite as a multifunctional cross-linker exhibit extraordinary mechanical toughness, tensile moduli and tensile strengths. ${ }^{16-20}$ But, the expensive price of Laponite is disadvantageous to industrial production on a large

*For correspondence scale. Therefore synthetic hectorite, acting as a physical cross-linker instead of traditional chemical crosslinkers, has been widely used to replace Laponite to form NC gels in order to decrease the cost. ${ }^{21-26}$

So far, almost all of the Laponite and hectorite-based $\mathrm{NC}$ gels are prepared by water solution polymerization. On the basis of our previous work on superabsorbent hydrogels and microemulsion polymerization, ${ }^{27-39}$ a commercially synthesized hectorite product was used to prepare NC gels by inverse microemulsion polymerization for future industrial production at a lower cost. In this study, effects of hectorite amount on water absorbency, gel strength and shear-resistance were investigated. Dynamic viscoelasticity behaviours of the nanocomposite hydrogels was also studied. Structure of the nanocomposite hydrogel was characterized by transmission electron microscope (TEM).

\section{Experimental}

\subsection{Materials}

Itaconic acid (IA) of analytical grade was purified by recrystallization. Acrylamide (AM) of analytical grade was purified by recrystallization. $\mathrm{N}$-isopropyl acrylamide (NIPAM) of analytical grade was purified by 
recrystallization. N-methylene-bis- acrylamide (MBA) of analytical grade was purified by recrystallization. Ammonium persulfate (APS), sodium bisulfite (SBS), sorbitan monooleate (Span 80), polyoxyethylene sorbi$\tan$ monooleate (Tween 80), cyclohexane of analytical grade were used without further purification.

\subsection{Synthesis of hectorite/ poly(AM/IA) nanocomposite hydrogels}

A series of nanocomposite hydrogels with different components were prepared by microemulsion polymerization according to the following procedure: Typically, $0.45 \mathrm{~g}$ hectorite was dispersed in $30 \mathrm{~mL}$ distilled water under stirring at least for $4 \mathrm{~h}$ to make a uniform dispersion. Then $2.25 \mathrm{~g}$ IA and desired amounts of $\mathrm{NaOH}$ solution were added into the hectorite aqueous dispersion in an ice bath. Subsequently $9 \mathrm{~g} \mathrm{AM}$ and 0.034 $\mathrm{g}$ MBA were added to the above aqueous dispersion under stirring at room temperature for $60 \mathrm{~min}$. Finally the mixed aqueous dispersion was added dropwise to the inverse microemulsions formed by $300 \mathrm{~g}$ cyclohexane, $18 \mathrm{~g}$ Span80 and $12 \mathrm{~g}$ Tween 80 . The water bath was heated slowly to $45-55^{\circ} \mathrm{C}$ with mild stirring under bubbling $\mathrm{N}_{2}$ gas after redox initiator APS (69 mg) and SBS (19 mg) were introduced to the above inverse microemulsions. After $3-5 \mathrm{~h}$ of the reaction, the resulting product was washed several times with ethanol and acetone, dried at $60^{\circ} \mathrm{C}$ to a constant weight, then milled and screened.

\subsection{Water absorbency of the nanocomposite hydrogels using filtration method}

Approximately $50 \mathrm{mg}$ of dried, milled and sifted hydrogels were dispersed in $100 \mathrm{~mL}$ of deionized water. Then, excess water was allowed to drain through a 300 mesh wire gauze. The weight of the hydrogels containing absorbed water was measured, and water absorbency at different absorption time was calculated according to the following equation:

$$
\text { Absorbency }(\mathrm{g} / \mathrm{g})=\left(W_{2}-W_{1}\right) / W_{1}
$$

where $W_{1}$ and $W_{2}$ are the weight of the dry and swollen hydrogels, respectively.

\subsection{Gel strength evaluation of the nanocomposite hydrogels}

The apparent viscosity was a relative measure of gel strength of swollen nanocomposite hydrogels and was determined on a NXS-11B type rotation viscometer
(Chengdu, China) at a shear rate of $17 \mathrm{~s}^{-1}$ at room temperature.

\subsection{Shear-resistance evaluation of the nanocomposite hydrogels}

Swollen nanocomposite hydrogels were agitated by a high-speed mixer for $60 \mathrm{~min}$. with a speed of $3000 \mathrm{rpm}$, and then gel strength of swollen nanocomposite hydrogels was determined on a NXS-11B type rotation viscometer (Chengdu, China) at a shear rate of $17 \mathrm{~s}^{-1}$ at room temperature.

\subsection{Dynamic viscoelasticity measurements}

Dynamic viscoelasticity measurements were performed with a Rheometric Fluids RFR 7800 rheometer. The applied frequency $(\omega)$ was changed from 0.1 to $10 \mathrm{rad} / \mathrm{s}$. The storage $\left(\mathrm{G}^{\prime}\right)$ and loss $\left(\mathrm{G}^{\prime \prime}\right)$ moduli were obtained from oscillatory measurements with stress of $50 \mathrm{~Pa}$, $100 \mathrm{~Pa}$ and $200 \mathrm{~Pa}$, respectively.

\subsection{Characterization of the nanocomposite hydrogels}

The micrographs of the nanocomposite hydrogels were taken using TEM (Tecnai G2 F20S-TWIN, FEI). Before TEM observation, inverse microemulsion samples were diluted with cyclohexane, stained with phosphotungstic acid, and then dipped onto the copper grid and allowed to evaporate at room temperature.

\section{Results and Discussion}

\subsection{Synthesis and morphology of the hectorite/poly(AM/IA) nanocomposite hydrogels}

A new facile strategy, as shown in scheme 1, is developed to synthesize hectorite/poly(AM/IA) nanocomposite hydrogels via microemulsion polymerization. Firstly partially neutralized IA, AM and MBA were added into the hectorite dispersion to form a water phase. Span80/Tween 80 used as complex surfactants were dissolved in cyclohexane to form an oil phase. Finally both phases were mixed together to form inverse microemulsion. The microemulsion polymerization was triggered by adding ammonium persulfate (APS) and sodium bisulfite (SBS) into the formed inverse microemulsion. Upon heating, the redox initiators will decompose to form primary anion radicals. These anion radicals can strongly interact with exfoliated hectorite clay platelets through ionic interactions 


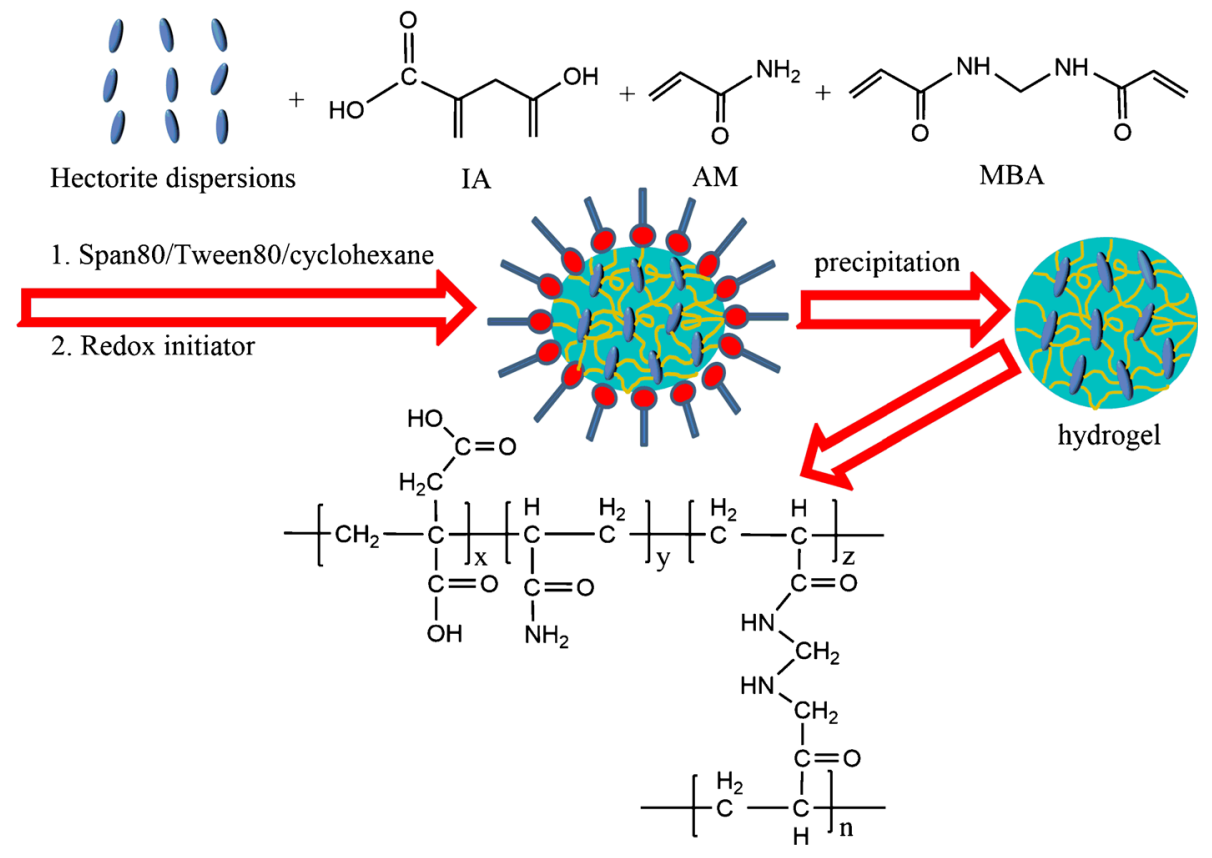

Scheme 1. Illustration of the formation of hectorite/poly(AM/IA) nanocomposite hydrogels via microemulsion polymerization.

and initiate the monomer on the hectorite clay surfaces, followed by chain propagation reaction. Finally, nanocomposite hydrogels with cross-linking network structure are formed with polymer chains attached to the hectorite clay platelets.

TEM image of the as-synthesized nanocomposite hydrogels was shown in figure 1 . The hydrogel particles were regular and spherical-like in shape, and had the average particle size of $43 \mathrm{~nm}$ in the range of 30-65 $\mathrm{nm}$, indicating that nanocomposite hydrogels had been successfully synthesized by inverse microemulsion polymerization.

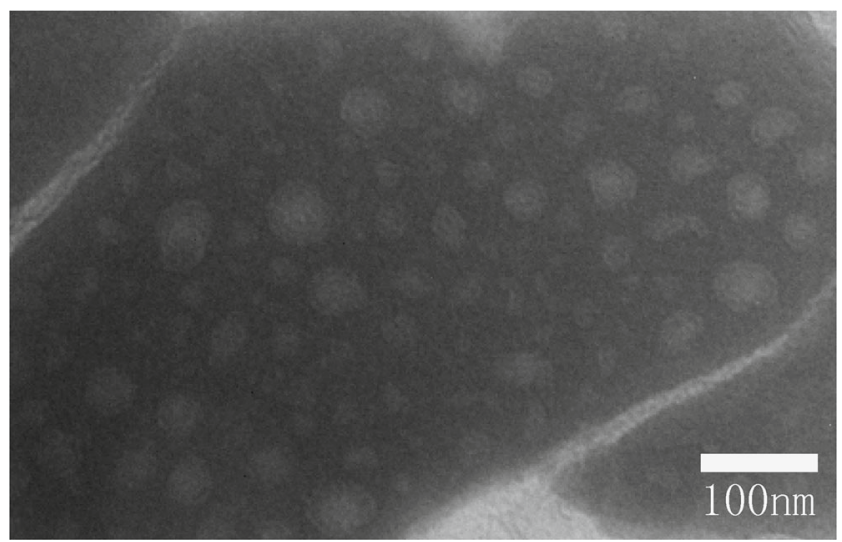

Figure 1. TEM image of the hectorite/poly(AM/IA) nanocomposite hydrogels.

\subsection{Effects of hectorite amount on the water} absorption of the nanocomposite hydrogels

Figure 2 plots the water absorbency rate of the nanocomposite hydrogels as a function of the hectorite amount. As shown in figure 2, the nanocomposite hydrogels absorbed water rapidly at the initial stage and then slowly with increasing absorption time, approaching equilibrium around $300 \mathrm{~min}$. Water absorbency decreased with increasing hectorite amount. Laponite,

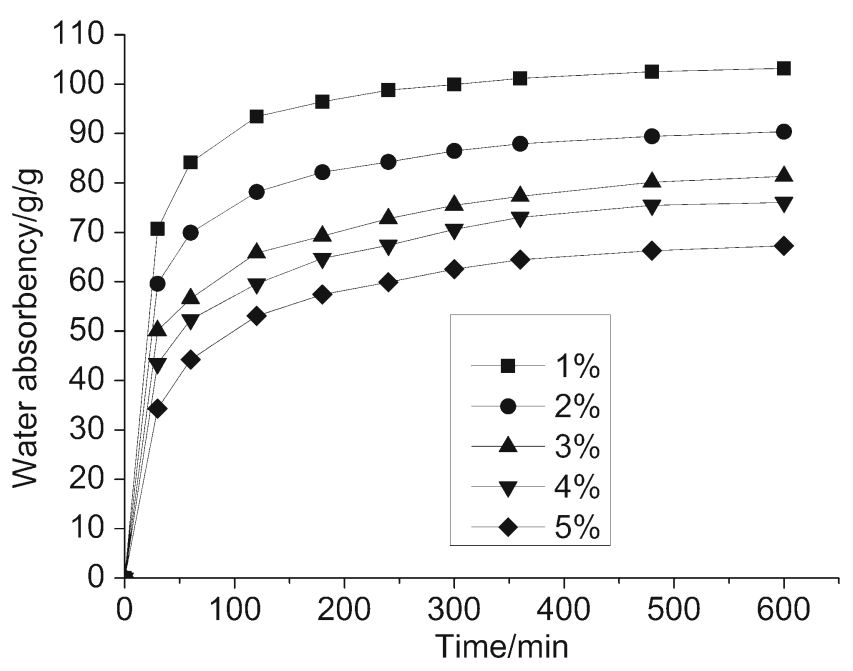

Figure 2. Effect of hectorite amount on the water absorbency rate of the hectorite/poly(AM/IA) nanocomposite hydrogels. 
a kind of hectorite clay, can act as a multifunctional cross-linker with a large effective functionality. ${ }^{12-15}$ The effective network chain density of the nanocomposite hydrogels increases with increasing hectorite clay amount due to an increase in the junction number formed by hectorite clay platelets. This can make the polymeric chains more rigid and restrain the expansion of network, thus decreasing water absorbency of the nanocomposite hydrogels.

\subsection{Effects of hectorite amount on the gel strength and shear-resistance of the nanocomposite hydrogels}

Figure 3 demonstrates the gel strength and shearresistance versus hectorite amount of the nanocomposite hydrogels. Gel strength increased with increasing hectorite amount and reached 9.1Pa.s at $5.0 \mathrm{wt} \%$ hectorite used. After $60 \mathrm{~min}$. shearing with a speed of $3000 \mathrm{rpm}$, gel strength of the nanocomposite hydrogels decreased, showing shear-thinning behaviours, which might be ascribed to the chain alignment and slippage of the macromolecules under high speed shearing, disturbing or destroying the hydrogen bonds between hectorite and amide and carboxylic groups of the macromolecular networks. However, gel strength of the nanocomposite hydrogels decreased more slightly after $60 \mathrm{~min}$. shearing with increasing hectorite amount, demonstrating enhanced shearresistance of the nanocomposite hydrogels. As a multifunctional cross-linker with a large effective functionality, more hectrite clay within the polymeric networks can increase the effective network chain density of the nanocomposite hydrogels. This can make the polymeric chains more rigid and restrain the deformation of

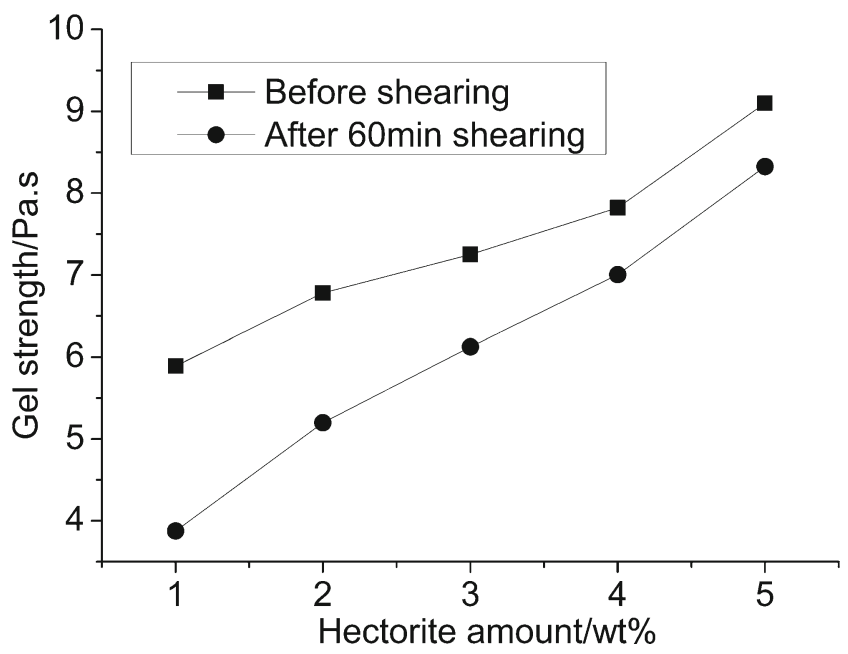

Figure 3. Effect of hectorite amount on the gel strength and shear-resistance of hectorite/poly(AM/IA) nanocomposite hydrogels. polymeric chains, thus increasing both gel strength and shear-resistance of the nanocomposite hydrogels.

\subsection{Dynamic rheological behaviours}

of the nanocomposite hydrogels

Rheology measurements under oscillating dynamic conditions were carried out on the swollen nanocomposite hydrogels, and the results were demonstrated in figure 4. Both storage moduli $\mathrm{G}^{\prime}$ and loss moduli $\mathrm{G}$ ? of the swollen nanocomposite hydrogels changed little with increasing oscillation frequency under stress of $50 \mathrm{~Pa}, 100 \mathrm{~Pa}$ and $200 \mathrm{~Pa}$, respectively. Predominantly solid-like behaviour (storage moduli $\mathrm{G}^{\prime}>$ loss moduli $\mathrm{G}$ ?) were observed in the whole oscillation frequency region, due to the three-dimensional network structure with both chemical cross-linking and physical crosslinking. $G^{\prime}$ decreased whereas $G^{\prime \prime}$ gradually increased with increased stress, an indicative of enhanced viscoelastic behaviours of the swollen nanocomposite hydrogels under high stress. Flexible polymeric chains
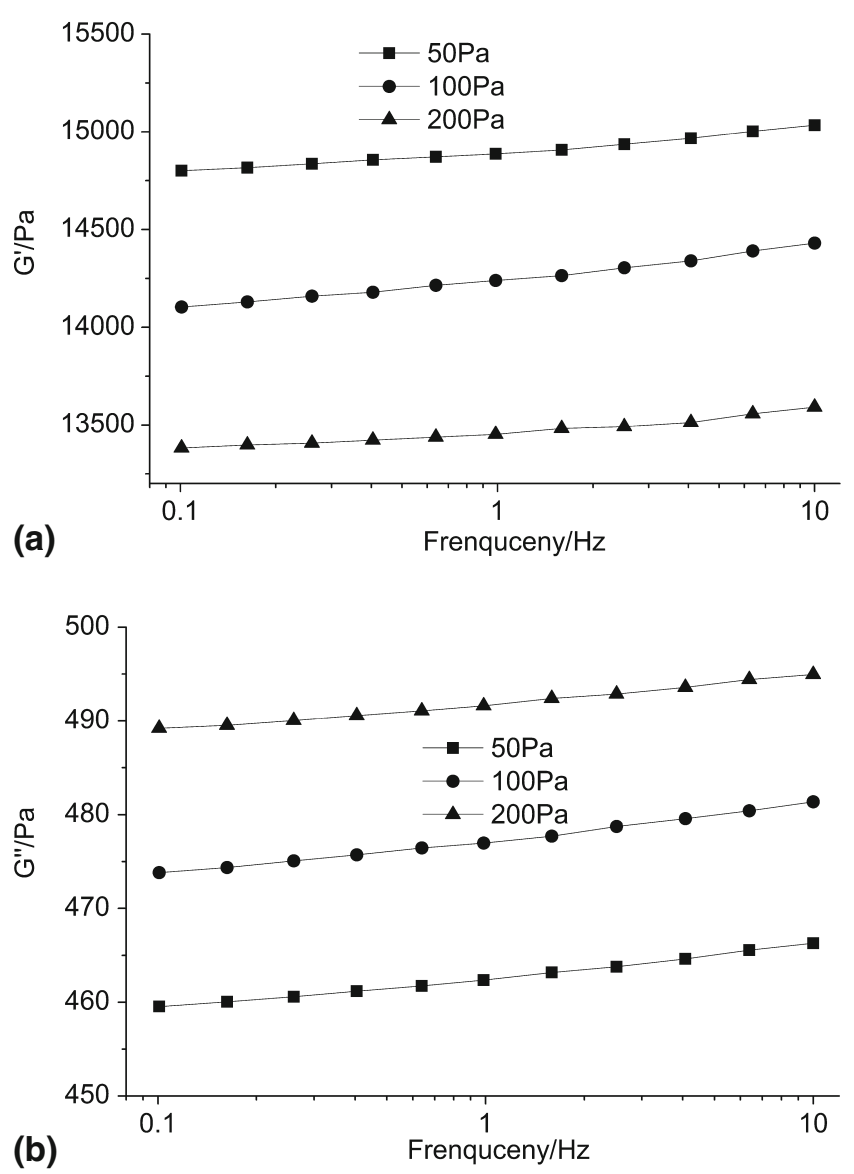

Figure 4. Variation of the storage modulus $G^{\prime}$ (a) and the loss modulus $G^{\prime \prime}$ (b) with the oscillation frequency for the hectorite/ poly(AM/IA) nanocomposite hydrogels at different stress. 
of the nanocomposite hydrogels are more liable to stretch twist and relax under high stress, resulting in an increasing liquid-like behaviour and decreasing solidlike behaviour. Therefore the nanocomposite hydrogels show remarkable viscoelastic behaviours under high stress.

\section{Conclusions}

A new hectorite/poly(AM/IA) nanocomposite hydrogel with high gel strength was synthesized by facile inverse microemulsion polymerization. Water absorbency decreased whereas gel strength and shear-resistance of the nanocomposite hydrogels increased with increased hectorite amount. The swollen nanocomposite hydrogels showed predominantly solid-like behaviour (storage moduli $\mathrm{G}^{\prime}>$ loss moduli $\mathrm{G}^{\prime \prime}$ ) in the whole oscillation frequency region and enhanced viscoelastic behaviours under high stress. TEM indicated that the as-synthesized regular and spherical hydrogel particles had the average particle size of $43 \mathrm{~nm}$ in the range of $30-65 \mathrm{~nm}$.

\section{Acknowledgements}

The authors are grateful to the support of PetroChina Innovation Foundation, China (2012D-5006-0212), Sichuan Provincial Science \& Technology Pillar Program, China (2013GZ0149, 2014GZX0010), Opening fund of State Key Laboratory of Geohazard Prevention and Geoenvironment Protection, Chengdu University of Technology, China (SKLGP2012K004) and the Sichuan youth science and technology innovation research team funding scheme, China (2013TD0005).

\section{References}

1. Kosemund K, Schlatter H, Ochsenhirt J L, Krause E L, Marsman D S and Erasala G N 2009 Regul. Toxicol. Pharm. 5381

2. Liang R, Yuan H B, Xi G X and Zhou Q X 2009 Carbohyd. Polym. 77181

3. Ibrahim S M, El Salmawi K M and Zahran A H 2007 J. Appl. Polym. Sci. 1042003

4. Duan J C, Lu Q, Chen R W, DuanY Q, Wang L F, Gao L and Pan S Y 2010 Carbohyd. Polym. 80436

5. Guilherme M R, Reis A V, Paulino A T, Fajardo A R, Muniz E C and Tambourgi E B 2007 J. Appl. Polym. Sci. 1052903

6. Sadeghi M and Hosseinzadeh H J 2008 J Bioact. Compat. Pol. 23381

7. Mahdavinia G R, Pourjavadi A, Hosseinzadeh $\mathrm{H}$ and Zohuriaan M J 2004 Eur. Polym. J. 401399

8. Chang C Y, Duan B, Cai J and Zhang L N 2010 Eur. Polym. J. 4692
9. Tongwa P, Nygaard R and Bai B J 2013 J. Appl. Polym. Sci. 128787

10. Hu J, Kurokawa T, Nakajima T, Sun T L, Suekama T, Wu Z L, Liang S M and Gong J P 2012 Macromolecules 459445

11. Hashmi S, Nejad A G, Obiweluozor F O, VatankhahVarnoosfaderani M and Stadler F J 2012 Macromolecules 459804

12. Okada K and Usuki A 2006 Macromol. Mater. Eng. 291 1449

13. Haraguchi $\mathrm{K}$ and Takehisa T 2002 Adv. Mater. 141120

14. Haraguchi K, Takehisa T and Fan S 2002 Macromolecules 3510162

15. Haraguchi K, Farnworth R, Ohbayashi A and Takehisa T 2003 Macromolecules 365732

16. Wu C J, Gaharwar A K, Chan B K and Schmidt G 2011 Macromolecules $\mathbf{4 4} 8215$

17. Wang Y R, Ma J H, Yang S G and Xu J 2011 Colloids Surf., A 39020

18. Hu X B, Xiong L J, Wang T, Lin Z M, Liu X X and Tong Z 2009 Polymer 501933

19. Xiong L J, Zhu M N, Hu X B, Liu X X and Tong Z 2009 Macromolecules $\mathbf{4 2} 3811$

20. Liu Y, Zhu M F, Liu X L, Zhang W, Sun B, Chen Y M and Adler H J P 2006 Polymer 471

21. Haraguchi K and Song L Y 2007 Macromolecules 40 5526

22. Kato N, Sakai Y and Shibata S 2003 Macromolecules 36961

23. Djonlagić J, Žugić D and Petrović Z 2012 J. Appl. Polym. Sci. 1243024

24. Ning J Y, Li G and Haraguchi K 2014 Macromol. Chem. Phys. 215235

25. Anbarasan R, Arvind $\mathrm{P}$ and Dhanalakshmi V $2011 \mathrm{~J}$. Appl. Polym. Sci. 121563

26. Mauroy $\mathrm{H}$, Plivelic $\mathrm{T}$ S, Hansen E L, Fossum J O, Helgesen G and Knudsen K D 2013 J. Phys. Chem. C 11719656

27. Wan T, Zhou Z L, Huang R Q, Zou C Z, Xu M, Cheng W Z and Li R X 2014 Appl. Clay Sci. 101199

28. Wan T, Li R X, Wu D Q, Hu Z W, Xu M, Cheng W Z and Zou C Z 2014 Polym. Bull. 712819

29. Wan T, Xiong L, Huang R Q, Zhao Q H, Tan X M, Qin L L and Hu J Y 2014 Polym. Bull. 71371

30. Wan T, Huang R Q, Zhao Q H, Xiong L, Luo L, Zhang H B and Cai G J 2014 J. Compos. Mater. 482341

31. Wan T, Huang R Q, Zhao Q H, Xiong L, Luo L, Tan X M and Cai G J 2013 J. Appl. Polym. Sci. 130698

32. Wan T, Huang R Q, Zhao Q H, Xiong L, Qin L L, Tan X M and Cai G J 2013 J. Appl. Polym. Sci. 1303404

33. Wan T, Yao J, Sun Z S, Wang L and Wang J $2011 \mathrm{~J}$. Petrol. Sci. Eng. 78334

34. Wan T, Zang T S, Wang Y C, Zhang R and Sun X C 2010 Polym. Bull. 65565

35. Wan T, Wu C, Ma X L, Yao J and Lu K 2009 Polym. Bull. 62801

36. Wan T, Yao J and Ma X L 2008 J. Appl. Polym. Sci. 110 3859

37. Wan T, Wang L, Yao J, Ma X L, Yin Q S and Zang T S 2008 Polym. Bull. 60431

38. Wan T, Wang X Q, Yuan Y and He W Q 2006 Polym. Int. 551413

39. Wan T, Wang X Q, Yuan Y and He W Q 2006J. Appl. Polym. Sci. 1022875 